\title{
Comparação entre métodos de estimativa de evapotranspiração potencial e de referência
}

\section{Comparison between potential and reference evapotranspiration estimation methods}

\author{
Brenda Mello Franco *1; Flávia Antunes Ziani *2; Júlia Konrad *3; Cácio Miranda Andres *4 \\ Aluno(a) de Graduação em Engenharia Sanitária e Ambiental, Universidade Federal de Santa Maria, Santa \\ Maria, Rio Grande do Sul, Brasil. \\ ${ }^{1}$ Orcid: http://orcid.org/0000-0002-4761-2915 E-mail: bremfr@gmail.com; \\ 2 Orcid: http://orcid.org/0000-0001-7325-6080 E-mail: eng.ziani@gmail.com; \\ 3 Orcid: http://orcid.org/0000-0002-2600-1023 E-mail: juliabkonrad@gmail.com; \\ ${ }^{4}$ Orcid: http://orcid.org/0000-0002-1667-2202 E-mail: cacio.mandres@gmail.com.
}

\begin{abstract}
RESUMO: Evapotranspiração é a variável mais ativa do ciclo hidrológico e a principal componente no balanço hídrico em ecossistemas agrícolas. É um parâmetro chave para estudos de avaliação ambiental e de manejo de bacias hidrográficas, para estimativa da necessidade hídrica das culturas e para projetos e manejo de sistemas de irrigação. O método de Penman-Monteith é o método indicado pela FAO devido a sua boa precisão. Já o método Thornthwaite é mais prático, devido a sua simplicidade de aplicação e o reduzido número de dados meteorológicos necessários. Nesse sentido, esse trabalho tem como objetivo comparar os valores de evapotranspiração de referência calculados por meio do método Penman-Monteith com os valores de evapotranspiração potencial calculados por meio do método Thornthwaite e avaliar as estimativas obtidas. A Evapotranspiração de Referência foi determinada através da aplicação do método Penman-Monteith-FAO sendo utilizados dados climatológicos de três estações meteorológicas do Instituto Nacional de Meteorologia: Santana do Livramento, Alegrete e Quaraí. Estes dados foram então comparados com os dados de Evapotranspiração Potencial do Atlas Climático da Fepagro, calculada pelo método Thornthwaite, e também com os dados de precipitação. Os resultados de comparação obtidos mostram que o método Thornthwaite, apesar de mais simplificado, mostrou bom ajuste com relação ao método PenmanMonteith, demonstrando que o método Thornthwaite pode ser usado para estimativa de dados de evapotranspiração em locais onde não há medição desse parâmetro ou que não há medição das variáveis meteorológicas necessárias à aplicação do método Penman-Monteith.
\end{abstract}

Palavras-chave: Bacia hidrográfica; Penman-Monteith; Thornthwaite.

ABSTRACT: Evapotranspiration is the most active variable in the hydrological cycle and the main component in the water balance in agricultural ecosystems. It is a key parameter for studies of environmental assessment and management of watersheds, for estimating the water requirements of crops and for irrigation systems projects and management. The Penman-Monteith method is the method indicated by FAO because of its good accuracy. The Thornthwaite method is more practical due to its simplicity of application and the reduced number of meteorological data requested. In this sense, the objective of this work is to compare the reference evapotranspiration values calculated using the Penman-Monteith method with the values of potencial evapotranspiration calculated using the Thornthwaite method and to evaluate the estimates obtained. The reference evapotranspiration was determined using the Penman-Monteith-FAO method, using climatological data from three meteorological stations of the National Meteorological Institute: Santana do Livramento, Alegrete and Quaraí. These data were then compared with the data of potential evapotranspiration of the Fepagro Climatic Atlas, calculated by the Thornthwaite method, as well as the precipitation data. The results show that the Thornthwaite method, although more simplified, showed a good fit in relation to the Penman-Monteith method, demonstrating that the Thornthwaite method can be used to estimate evapotranspiration data in places where there is no measurement of this parameter or that there is no measurement of the meteorological variables necessary for the application of the Penman-Monteith method.

Keywords: Watersheds; Penman-Monteith; Thornthwaite.

DOI 10.18554/rbcti.v4i2.3715 


\section{INTRODUÇÃO}

O balanço hídrico climatológico é uma das informações básicas para o planejamento racional das atividades agrícolas e florestais. Esta técnica consiste em contabilizar a evapotranspiração com a precipitação, considerando-se uma determinada capacidade de armazenamento de água no solo, de forma a determinar a disponibilidade de água às culturas, nas diferentes épocas do ano e a melhor estação de cultivo para uma determinada região (D'ANGIOLELLA; DA SILVA, 2004).

A evapotranspiração (ET) é a variável mais ativa do ciclo hidrológico e a principal componente no balanço hídrico em ecossistemas agrícolas. Portanto, ela é um parâmetro chave para estudos de avaliação ambiental e de manejo de bacias hidrográficas (RAZIEl; PEREIRA, 2013), para a estimativa da necessidade hídrica das culturas e para projetos e manejo de sistemas de irrigação (KUMAR et al., 2008).

No final da década de 1940, duas importantes contribuições científicas surgiram para aprimorar não só a agricultura, mas, também, a climatologia e hidrologia: os trabalhos de Warren Thornthwaite (1948) e de Howard Penman (1948). O maior objetivo de Thornthwaite foi explicar as variações sazonais do balanço de água no solo e tentar diferenciar as diferenças climáticas regionais e, por isso, este método é apenas em função da temperatura média do ar e da duração do dia, em média mensal. Já Penman deu enfoque aos processos físicos envolvidos na evaporação e resultou no desenvolvimento de um método, que, a partir de fatores meteorológicos, expressa a estimativa da taxa de evaporação da água em contato com a atmosfera, da umidade da superfície do solo e da vegetação. Ou seja, sua análise científica foi baseada no conhecimento físico que rege o fenômeno (SEDIYAMA, 1996; CAMARGO; CAMARGO, 2000).

A partir desses trabalhos, surgiu o conceito de evapotranspiração potencial (ETp), caracterizada como a transferência de água do sistema solo-planta para a atmosfera, sob condições padronizadas. Ou seja, uma área com extensa superfície natural, coberta por vegetação baixa, uniforme e de elevado índice de área foliar, com um alto crescimento na sua fase adulta, sendo a grama o tipo de vegetação mais utilizado, e teor de água no solo próximo ou na capacidade de campo. Nessas condições de contorno, e considerando-as invariáveis no tempo e espaço, a transferência de água do sistema solo-planta para a atmosfera (evapotranspiração) ocorre nas condições atmosféricas na vegetação, sem interferências advectivas, podendo ser estimada por métodos com embasamentos matemáticos teórico-empíricos desenvolvidos e testados para várias condições climáticas (CARVALHO et al., 2011).

Na década de 1960, Monteith, com base no método de Penman, propôs um novo método que estimava diretamente a ET, denominando-o de Penman-Monteith, e Doorenbos e Pruitt (1977) introduziram um novo conceito de evapotranspiração de referência (ETo), em substituição ao termo ETp. Mas foi em 1990 que houve uma grande revolução sobre a proposição da conceituação e estimativa da ETo. Neste ano, a Food and Agriculture Organization (FAO) promoveu, em Roma, Itália, um encontro de especialistas na área, para atender a vários objetivos, dentre eles o de analisar os conceitos e procedimentos de metodologias de cálculos da evapotranspiração, com enfoque no estabelecimento de uma nova definição para a cultura de referência e o 
método que pudesse estimar a evapotranspiração, para esta referência (PEREIRA et al. 1997).

Assim, o novo conceito proposto para a ETp passou a ser, de fato, a evapotranspiração de referência (ETo), tornando-se, este conceito, desde então, mais utilizado e o método recomendado que, após parametrização, passou a denominar-se Penman-Monteith FAO, o qual foi amplamente aceito, internacionalmente.

Neste caso, a referência utilizada é de uma cultura hipotética, cujas características se assemelham, à ET da grama. Com isto pode se permitir que tais características (valores numéricos) mantenham-se como parâmetros constantes adotados no cálculo da ETo. Estes parâmetros, para a cultura hipotética, são: altura de 0,12 m, albedo igual a 0,23 e resistência da cultura ao transporte de vapor d'água igual a $69 \mathrm{~s} \mathrm{m-1}$. Logo, a ETo é um elemento indicativo da demanda hídrica das culturas de um determinado local e período (ALLEN et al., 1998).

Apesar da proposição da FAO, ainda é comum o uso dos termos ETp e ETo. Podese inferir que, para estudos climatológicos, o termo ETp continue sendo o mais utilizado, pois, quase sempre, está ligado à análise do potencial hídrico de uma região, enquanto a ETo é bastante adequada para projetos e manejo de irrigação, uma vez que a ETc, normalmente, é determinada em duas etapas, aplicando-se o coeficiente de ajuste Kc à evapotranspiração da cultura de referência (ETo), que, no caso, é a cultura hipotética (CARVALHO et al., 2011).

Nota-se, entretanto, que para a utilização da equação de Penman-Monteith há necessidade de se obter algumas variáveis meteorológicas que podem não estar disponíveis no local de sua aplicação, além do extenso processo de cálculo envolvido. Nesse sentido, apesar do método de Thornthwaite apresentar certa limitação na estimação, podendo resultar em sobre ou subestimação dos valores, o mesmo é mais prático, tendo em vista a sua simplicidade de aplicação e o reduzido número de dados meteorológicos necessárias (BROCHET; GERBIA, 1975).

Nesse sentido, esse trabalho tem como objetivo comparar os valores de ETo calculados por meio do método de Penman-Monteith com os valores de ETp calculados por meio do método de Thornthwaite com dados de três estações meteorológicas situadas na região da bacia hidrográfica do rio Ibirapuitã.

\section{MÉTODO}

A área de estudo escolhida foi a bacia hidrográfica do Rio Ibirapuitã. Esta bacia foi escolhida como área de estudo devido a ser uma região de grande produção orizícola, de forma que o principal uso da água nesta bacia se destina a irrigação, o que pode ocasionar insuficiência hídrica nos meses de verão (SEMA, 2019), e devido a conter a maior Unidade de Conservação do Bioma Pampa no Brasil, a Área de Proteção Ambiental (APA) do Ibirapuitã, importante área de proteção da biodiversidade e da beleza paisagística da bacia hidrográfica do rio Ibirapuitã (SILVA, 2011).

O rio Ibirapuitã corresponde a um dos principais tributários da margem esquerda do rio Ibicuí, maior e principal rio que compõem a chamada região hidrográfica do rio Uruguai (SEMA, 2019). A bacia hidrográfica do Ibirapuitã (Figura 1) é de $7^{\mathrm{a}}$ ordem, com área da bacia correspondente a $7.978,70 \mathrm{~km}^{2}$ e o perímetro $646.120 \mathrm{~km}$. A extensão do canal principal é de $597,72 \mathrm{~km}$, que escoa de sul para norte, formando um padrão de drenagem retangular controlado pelas estruturas presentes nas rochas vulcânicas do substrato 
geológico. A bacia hidrográfica do rio Ibirapuitã, do médio ao baixo curso, drena áreas do município de Alegrete (MENEZES; TRENTIN, 2017).

Figura 1. Mapa de localização da Bacia Hidrográfica do rio Ibirapuitã e das estações meteorológicas do INMET utilizadas no estudo.
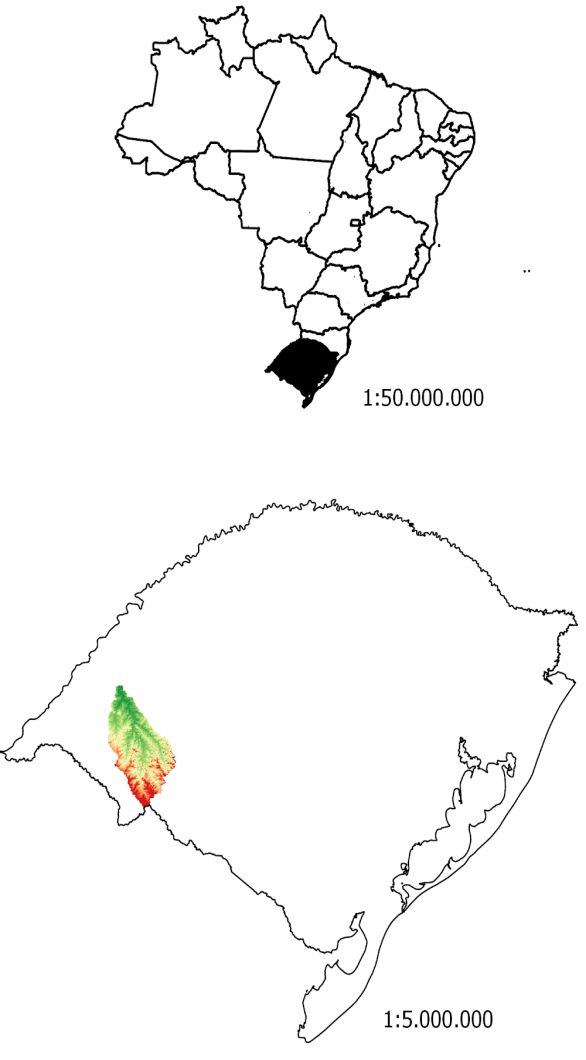

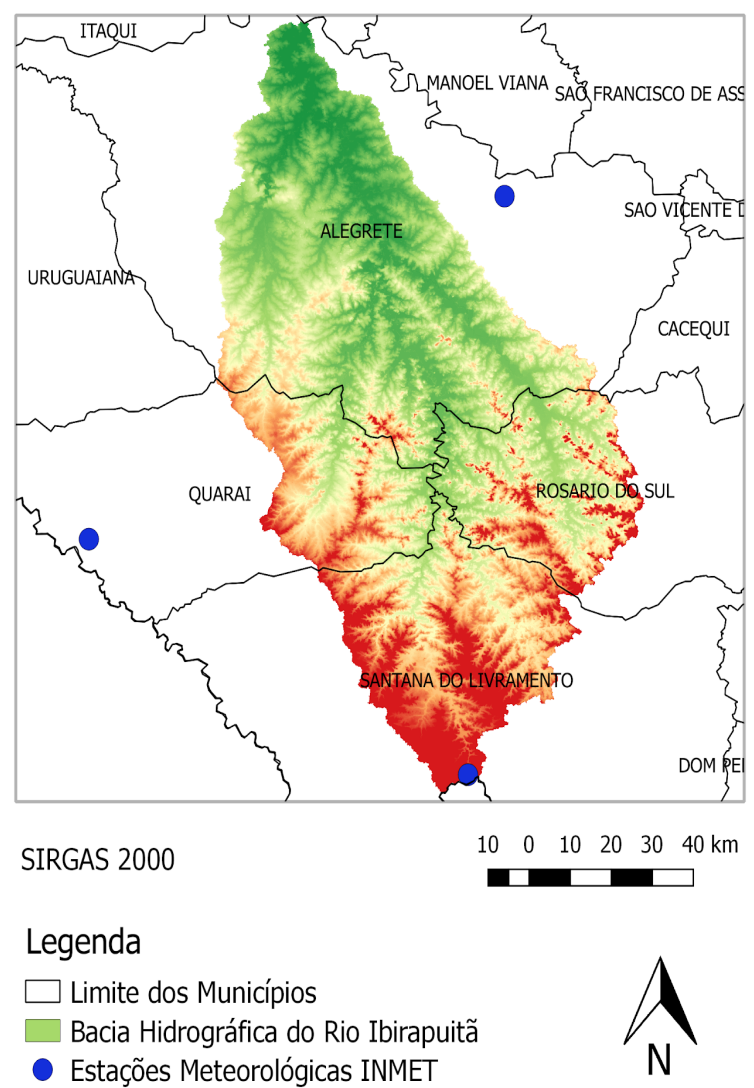

- Estações Meteorológicas INMET

A Evapotranspiração de Referência foi determinada através da aplicação do método de Penman-Monteith-FAO, segundo descrito na Circular Técnica 65 da Embrapa (EMBRAPA, 2006). Para tal, foram utilizados dados climatológicos de três estações meteorológicas do Instituto Nacional de Meteorologia (INMET): Santana do Livramento (83953), Alegrete (86975) e Quaraí (86992). A estação de Santana do Livramento está localizada dentro da área de estudo, enquanto as estações de Alegrete e Quaraí distam da área de estudo cerca de $13 \mathrm{Km}$ e $40 \mathrm{~km}$, respectivamente. Os dados históricos das estações foram solicitados diretamente ao INMET. Ressalta-se que para o cálculo foram utilizados apenas meses sem falhas nos dados.

Estes dados foram então comparados com os dados de Evapotranspiração Potencial do Atlas Climático da Fepagro, calculada pelo método de Thornthwaite, e também com os dados de precipitação (MATZENAUER et al., 2011). A comparação se deu pela determinação do método de Penman-Monteith como padrão e cálculo do coeficiente de determinação $\left(R^{2}\right)$ com os dados obtidos do método de Thornthwaite. Dessa forma, é possível avaliar a qualidade do ajuste e a variabilidade dos resultados obtidos a partir do método de Thornthwaite com Penman-Monteith para as estações consideradas. 
A análise dos dados foi feita de acordo com o ano hidrológico para a região, o qual corresponde a um período fixo de 12 meses, a começar no início do período chuvoso e terminar no final da estação seca. Em regiões com sazonalidade não tão evidente, como o Rio Grande do Sul, bibliografias sugerem o emprego do ano hidrológico de maio a abril (NAGHETTINI; PINTO, 2007; KICH; MELATI; MARCUZZO, 2015).

\section{RESULTADOS E DISCUSSÃO}

Na Tabela 1, tem-se os valores encontrados de ETo e ETp para os métodos de Penman-Monteith e Thornthwaite, respectivamente, para a Estação de Alegrete/RS.

Tabela 1. Resultados de ETo para o método de Penman-Monteith e ETp para o método de Thornthwaite, para a Estação de Alegrete/RS

\begin{tabular}{ccccccccccccc}
\hline Data & Mai & Jun & Jul & Ago & Set & Out & Nov & Dez & Jan & Fev & Mar & Abr \\
\hline $\begin{array}{c}\text { ETp } \\
\begin{array}{c}\text { Thornthwaite } \\
(\mathrm{mm})\end{array}\end{array}$ & 45 & 32 & 32 & 45 & 55 & 70 & 120 & 140 & 150 & 120 & 110 & 70 \\
\hline $\begin{array}{c}\text { ETo Penman- } \\
\text { Monteith (mm) }\end{array}$ & 41,4 & 31,1 & 38,3 & 60,2 & 64,2 & 82,1 & 101,5 & 110,9 & 112,9 & 88,5 & 79,9 & 58,8 \\
\hline $\begin{array}{c}\text { Precipitação } \\
\text { (mm) }\end{array}$ & 110,8 & 112,5 & 99,5 & 67 & 134,9 & 159,8 & 129,4 & 102,5 & 140,5 & 160,2 & 155,1 & 225,7 \\
\hline
\end{tabular}

Na Figura 2, com base nos valores encontrados na Tabela 1, tem-se o gráfico de média mensal de um ano hidrológico onde consta a ETo resultante para o método de Penman-Monteith e ETp para o método de Thornthwaite em relação a precipitação de cada mês para cidade de Alegrete/RS.

Figura 2 - Relação mensal entre Precipitação e Evapotranspiração Potencial para a Estação de Alegrete/RS.

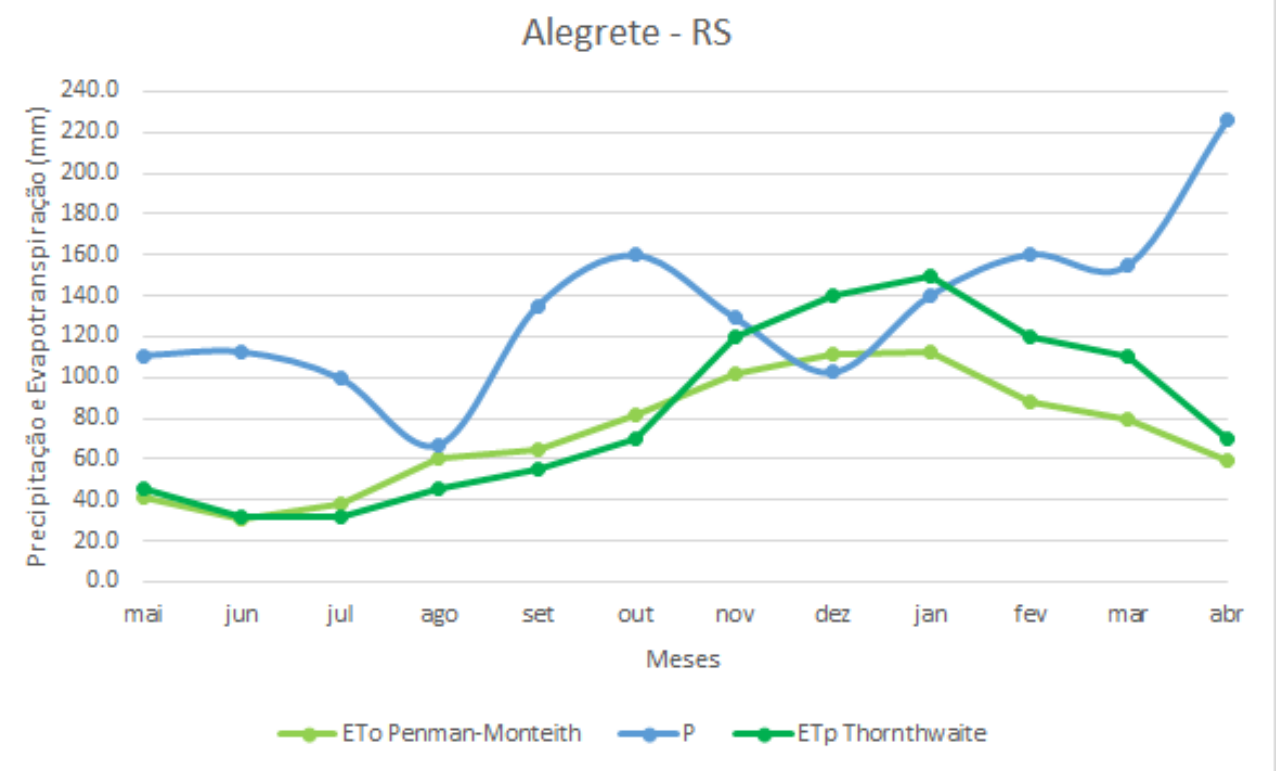


Analisando o gráfico da Figura 2, e os resultados apresentados na Tabela 1, notase que, os resultados do método de Penman-Monteith se mantém mais equilibrados quando comparados aos resultados do método de Thornthwaite, os quais apresentam dois picos, um de baixa ETp nos meses de junho e julho e outro de alta nos meses de dezembro e janeiro.

Para a estação de Santana do Livramento/RS os valores de ETo e ETp para os métodos de Penman-Monteith e Thornthwaite respectivamente, encontram-se na Tabela 2.

Tabela 2. Resultados de ETo para o método de Penman-Monteith e ETp para o método de Thornthwaite, para Estação de Santana do Livramento/RS.

\begin{tabular}{cccccccccccccc}
\hline Data & Mai & Jun & Jul & Ago & Set & Out & Nov & Dez & Jan & Fev & Mar & Abr \\
\hline $\begin{array}{c}\text { ETp Thornthwaite } \\
(\mathrm{mm})\end{array}$ & 41,3 & 28,5 & 28,5 & 37,9 & 45,1 & 58,4 & 88,2 & 119 & 129,5 & 103,7 & 95,1 & 61 \\
\hline $\begin{array}{c}\text { ETo Penman- } \\
\text { Monteith (mm) }\end{array}$ & 55,82 & 42,4 & 47,9 & 64,13 & 62,7 & 79,4 & 101,3 & 118,2 & 122,7 & 101,1 & 100,4 & 77,2 \\
\hline \begin{tabular}{c} 
Precipitação (mm) \\
\hline
\end{tabular} & 160 & 120 & 120 & 80 & 140 & 160 & 140 & 140 & 140 & 160 & 140 & 200 \\
\hline
\end{tabular}

A Figura 3 permite análise do comportamento da ETo e ETp citadas na Tabela 2.

Figura 3 - Relação mensal entre Precipitação e Evapotranspiração Potencial para a Estação de Santana do Livramento/RS.

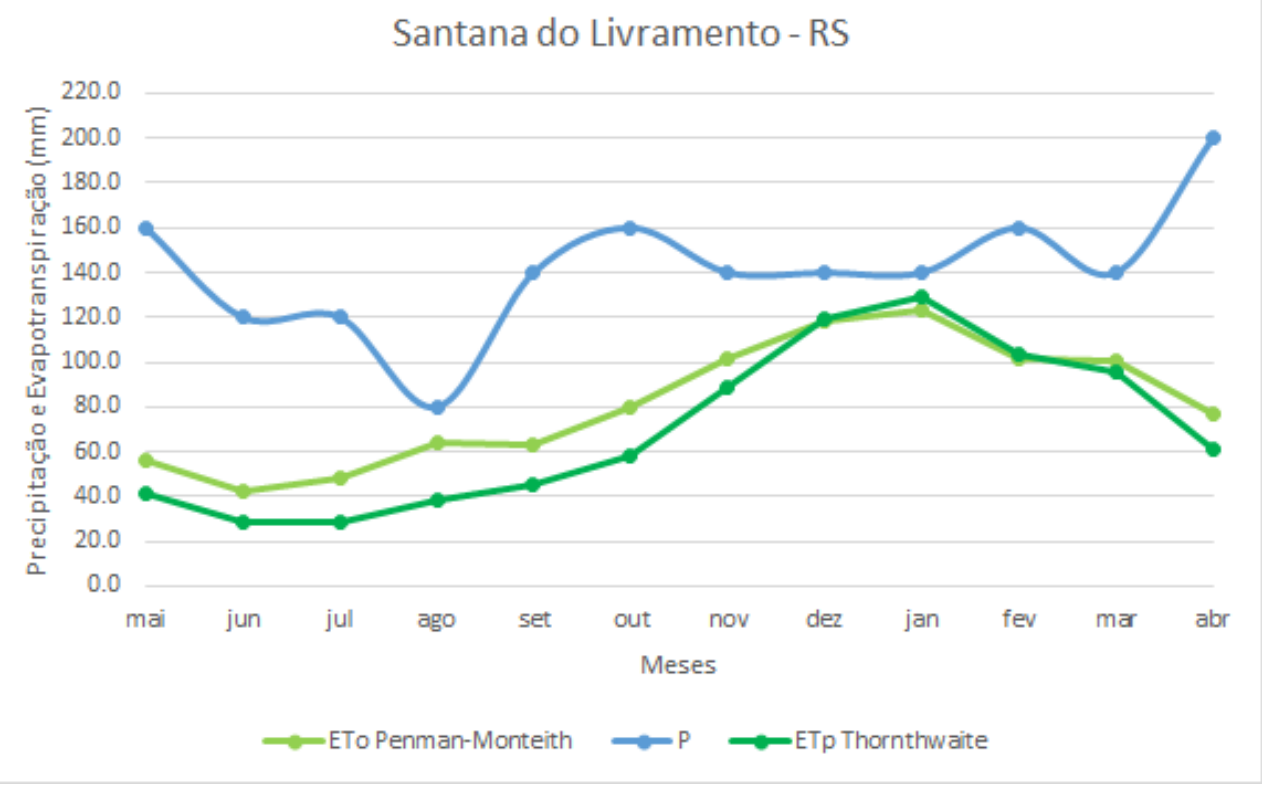

A Figura 3 e a Tabela 2, contém os valores de ETo estimados pelo método de Penman-Monteith mais equilibrados do que os valores de ETp estimados por Thornthwaite. Porém nos meses de verão os valores dos dois métodos encontram-se mais próximos que nos meses de inverno, os quais apresentam certa discrepância. Ainda, outro fato que difere a Figura 2 é a não existência de déficit hídrico, na qual a evapotranspiração é maior do que a precipitação. 
Na Tabela 3, tem-se os valores de precipitação e os resultados dos métodos de Thornthwaite e Penman-Monteith para a Estação de Quaraí/RS.

Tabela 3 - Resultados de ETo para o método de Penman-Monteith e ETp para o método de Thornthwaite para Estação de Quaraí/RS.

\begin{tabular}{cccccccccccccc}
\hline Data & Mai & Jun & Jul & Ago & Set & Out & Nov & Dez & Jan & Fev & Mar & Abr \\
\hline $\begin{array}{c}\text { ETp Thornthwaite } \\
(\mathrm{mm})\end{array}$ & 45 & 30 & 32 & 45 & 50 & 65 & 110 & 140 & 150 & 120 & 110 & 70 \\
\hline $\begin{array}{c}\text { ETo Penman- } \\
\text { Monteith (mm) }\end{array}$ & 41,8 & 35,3 & 46,8 & 67,3 & 67,6 & 78,2 & 99,4 & 121,4 & 117,9 & 97,6 & 82,2 & 62,7 \\
\hline $\begin{array}{c}\text { Precipitação } \\
(\mathrm{mm})\end{array}$ & 130,2 & 92,6 & 83,7 & 73,9 & 96,9 & 140,6 & 138,9 & 122,2 & 138,2 & 155,8 & 140, & 182,6 \\
& & & & & & & & & & & 5 & \\
\hline
\end{tabular}

Na Figura 4, tem-se o gráfico da Estação de Quaraí/RS, com base nos valores da Tabela 3.

Figura 4 - Relação mensal entre Precipitação e Evapotranspiração Potencial para a Estação de Quaraí/RS.

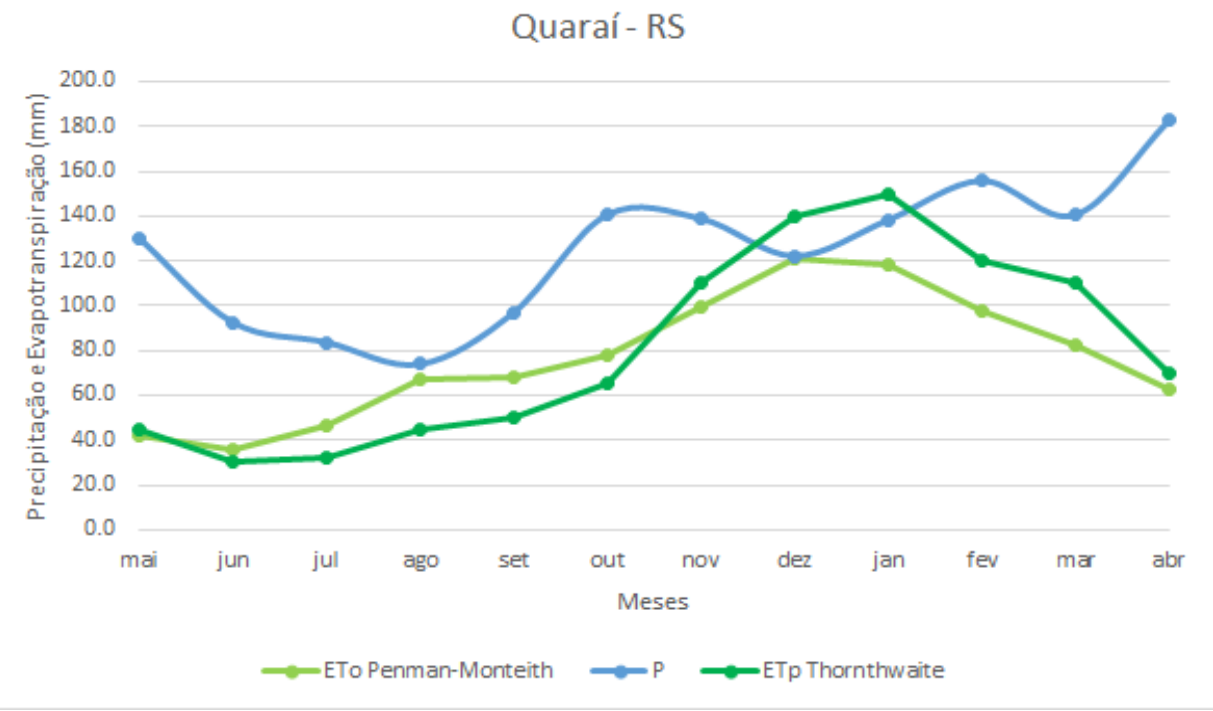

Para a cidade de Quaraí/RS, o gráfico presente na Figura 4 apresenta resultados semelhantes ao gráfico da Figura 2, para cidade de Alegrete/RS.

Com os resultados expressos, observa-se que os valores de evapotranspiração estimados por ambos os métodos apresentam a mesma tendência nas três estações estudadas. Entretanto, nota-se que a evapotranspiração estimada pelo método de Thornthwaite apresenta maior amplitude (diferença de extremos) se comparada com a evapotranspiração estimada pelo método de Penman-Monteith, o que já era esperado, 
pois conforme Brochet e Gerbia (1975), o método de Thornthwaite pode apresentar sobre e subestimação dos valores.

Ainda, o método de Thornthwaite apresentou ocorrência de déficit hídrico nos meses de verão (dezembro e janeiro), demonstrando que nesse período a precipitação que ocorre na região não é suficiente para suprir a demanda hídrica de referência das plantas.

De acordo com Allen et al. (1998), o método indicado como padrão é o de PenmanMonteith, dessa forma, usou-se o mesmo como referência neste estudo. Com os resultado do método de Thornthwaite, calculou-se o coeficiente de determinação, $R^{2}$, para avaliação do ajuste dos dados. Entre as três estações analisadas, a que mais o método de Thornthwaite se aproximou do padrão, foi a de Santana do Livramento (0.96). Nas estações de Alegrete e Quaraí, o método mostrou um ajuste bom, porém ligeiramente menor (0.89 em ambas).

Esses resultados indicam que o método de Thornthwaite pode ser usado para estimar a evapotranspiração nessas estações. Porém, como o mesmo faz uso de apenas a variável meteorológica da temperatura, deve ser usado com cautela e comparado com outro método mais preciso.

\section{CONCLUSÕES}

Observa-se que os valores de evapotranspiração estimados por ambos os métodos apresentam a mesma tendência nas três estações estudadas. Entretanto, nota-se que a evapotranspiração estimada pelo método de Thornthwaite apresenta maior amplitude (diferença de extremos) se comparada com a evapotranspiração estimada pelo método de Penman-Monteith.

Os resultados obtidos mostram que o método de Thornthwaite, apesar de ser mais simplificado, mostrou um bom ajuste com relação ao método de Penman-Monteith. Os dados de evapotranspiração mostraram-se próximos, um método de outro, o que pode ser confirmado por meio do coeficiente de determinação, o qual inclusive teve um ótimo resultado de 0.96 para uma das estações estudadas.

Conclui-se que o método de Thornthwaite pode ser usado para estimativa de dados de evapotranspiração em locais os quais não há medição desse parâmetro ou que não há medição das variáveis meteorológicas necessárias à aplicação do método de Penman-Monteith. A simplicidade deste método está fundado no fato de se utilizar somente da variável temperatura para cálculo e, dessa forma, deve ser comparado com métodos mais robustos e precisos, em estudos e trabalhos os quais envolvam a estimativa da evapotranspiração, para determinar sua precisão e aplicabilidade.

\section{REFERÊNCIAS}

ALLEN, R.G.; PEREIRA, L.S.; RAES, D.; SMITH, M. Crop evapotranspiration: guidelines for computing crop water requirements. FAO - Irrigation and drainage paper, 56, 1998.

BROCHET, P.; GERBIER, N. L'Evapotranspiration. In: Annales de Géographie, Paris 1975. 
CAMARGO, A. P.; CAMARGO, M. B. P. Uma revisão analítica da evapotranspiração potencial. Bragantia, Campinas, v. 59, n. 2, p. 125-137, 2000.

CARVALHO, D. F. DE; SILVA, D. G. DA; SOUZA, A.P. DE; GOMES, D. P.; ROCHA, H. S. da. Coeficientes da equação de Angström-Prescott e sua influência na evapotranspiração de referência em Seropédica, RJ. Revista Brasileira de Engenharia Agrícola e Ambiental, v.15, p.838-844, 2011.

D'ANGIOLELLA, G.; SILVA, J. Balanço Hídrico Climatológico do Brasil. In: 2004 XIII Congresso Brasileiro de Meteorologia. Fortaleza, 2004.

DOORENBOS, J.; PRUITT, W. O. Crop water requirements. Rome: FAO- Irrigation and drainage paper, 24, 1977.

EMBRAPA. Roteiro de cálculo da evapotranspiração de referência pelo método de Penman-Monteith-FAO. Bento Gonçalves, RS. Circular Técnica 65 da Embrapa, 2006.

$\mathrm{KICH}$, E. DE M.; MELATI, M. D.; MARCUZZO, F. F. N. Estudo do regime hídrico pluvial e fluvial na sub-bacia 86 visando a determinação do seu ano hidrológico. In: XXI Simpósio Brasileiro de Recursos Hídricos. Brasília, 2015.

KUMAR, M.; BANDYOPADHYAY, A.; RAGHUWANSHI, N.S.; Singh, R. Comparative study of conventional and artificial neural network-based ETo estimation models. Irrigation Science, v.26, p.531-545, 2008.

MATZENAUER, RONALDO; RADIN, BERNADETE; ALMEIDA, IVAN RODRIGUES De (Ed.). Atlas Climático: Rio Grande do Sul. Porto Alegre: Secretaria da Agricultura Pecuária e Agronegócio; Fundação Estadual de Pesquisa Agropecuária (FEPAGRO), Porto Alegre, 2011.

MENEZES, D; TRENTIN, R. Caracterização Morfométrica Da Bacia Hidrográfica Do Rio Ibirapuitã - RS. In: 2017 I Congresso Nacional de Geografia Física, Campinas, SP, 2017.

NAGHETTINI, M.; PINTO, E. J. A. Hidrologia Estatística. CPRM. Belo Horizonte, v.561, n.1, p. 552, 2007.

PENMAN, H. L. Natural evaporation from open water, bare soil, and grass. Proceedings of the Royal Society, London, v. 193, n. 1, p. 120-146, 1948.

PEREIRA, A. R.; VILLA NOVA, N. A.; SEDIYAMA. G. C. Evapo(transpi)ração. Piracicaba: Fealq. Embrapa, p. 183, 1997.

RAZIEI, T; PEREIRA, L.S. Estimation of ETo with Hargreaves-Samani and FAO-PM temperature methods for a wide range of climates in Iran. Agricultural Water Management, v.121, p.1-18, 2013.

SEDIYAMA, G. C. Estimativa da evapotranspiração: histórico, evolução e análise crítica. Revista Brasileira de Agrometeorologia, Santa Maria, v. 4, n. 1, p. 1-7, 1996. 
SEMA - Secretaria Estadual do Meio Ambiente. 2019. U050 - Bacia Hidrográfica do Rio Ibicuí. Porto Alegre, RS. Disponível em: https://www.sema.rs.gov.br/u050-baciahidrografica-do-rio-ibicui. Acesso em: 05 fev. 2019.

SILVA, E. L. da. O Pampa e a APA do Ibirapuitã: Estratégias e Ações para a Sustentabilidade. Programa de Pós graduação em Geografia UFSM, 2011. Disponível em: <http://w3.ufsm.br/ppggeo/files/ebook02/Artigo\%202.pdf> Acesso em: 20 mai 2019.

THORNTHWAITE, C. W. An approach towards a rational classification of climate. Geographycal Review, London, v. 38, n. 1, p. 55-94, 1948.

Recebido em: 08/05/2019

Aprovado em: 24/06/2019 